\title{
PENSAR LA EPISTEMOLOGÍA DEL TRABAJO SOCIAL
}

\author{
OCTAVIO VÁZQUEZ AGUADO \\ Trabajador Social. Antropólogo. \\ Profesor Titular de Escuela Universitaria. Departamento de Sociología y Trabajo Social. \\ Universidad de Huelva.
}

\section{ABSTRACT}

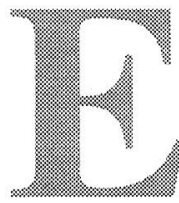

n el presente trabajo realizamos una reflexión a cerca de la epistemología del trabajo social. Partimos de considerar la epistemología como una reflexión de "segundo orden" frente a las producciones de la disciplina y profesión, siendo importante analizar qué relaciones mantiene con los contextos sociales donde se producen. En nuestro caso, vemos cuáles son los marcadores que más influyeron en el nacimiento del trabajo social y sus repercusiones en la consideración social de la disciplina. Creemos que la epistemología del trabajo social ha de hacerse teniendo en cuenta las aportaciones de este debate en el conjunto de las ciencias sociales, del cual nos interesa destacar lo que se denomina la concepción ampliada o nueva de las ciencias humanas, donde las características particulares del trabajo social encuentran la justificación para poder generar conocimiento. Establecido ya lo que nos aportan las ciencias sociales, abordamos el análisis de nuestra disciplina centrándonos en su concepto, objeto y método. Creemos que es posible la construcción de conocimientos puesto que, en las intervenciones del trabajo social, están presentes los grandes problemas teóricos de las ciencias sociales. Establezcamos una mínima distancia sobre las mismas para poder reflexionar sobre ellas.

\section{INTRODUCCIÓN}

El ser humano vive inserto en un medio físico y social determinado. A lo largo del espacio y del tiempo, ha intentado conocer ese medio de diversas formas y utilizando diferentes procedimientos, cada uno de los cuales le ha proporcionado una explicación concreta sobre la realidad. Esta explicación ha nutrido el capital de conocimientos que ha ido acumulando. A veces, sumativamente; otras veces, una explicación ha sustituido a la anterior. El conocimiento es pues unạ posibilidad y ne- 
cesidad que todas las sociedades han desarrollado de una u otra forma. En nuestro contexto, en el que se ubica el trabajo social, el conocimiento científico es el instrumento que nos hemos dado para señalar la diferencia entre lo cierto y lo falso

Nadie puede dudar de la importancia de la ciencia en nuestra sociedad. El desarrollo de la misma está en la base de la organización y de la vida social. Hay pues que interrogarse por sus repercusiones en todos los ámbitos de la sociedad. El trabajo social, en cuanto que está presente en una sociedad donde la ciencia es el criterio dominante para el establecimiento de lo verdadero y de lo falso, tiene que preguntarse por su carácter científico o no. La epistemología será un medio para ello. La concepción que tenemos sobre la misma vendría determinada por considerar que consiste en un análisis de las estructuras conceptuales de una ciencia en particular y de la ciencia en general. Este análisis se ubica en un nivel de segundo orden con respecto a la reflexión científica misma. Su objeto de trabajo vendría determinado no por entidades acotadas espacio-temporales..., sino por los conceptos que manejan los especialistas de esta ciencia para el desarrollo de la misma (Ulises Moulines, 1988). La epistemología no quiere imponer un sistema a priori, dogmático, que dicte autoritariamente lo que debe ser conocimiento científico, sino estudiar la génesis y la estructura de los conocimientos científicos, es decir, estudiar la producción científica desde todos sus aspectos, sin obviar que los conceptos empleados, y la misma ciencia, se producen en un contexto determinado por lo que habría que analizar la relación entre ciencia y sociedad (Mardones y Ursúa, 1982: 41-44).

\section{POR QUÉ UNA EPISTEMOLOGÍA DEL TRABAJO SOCIAL}

Para contestar esta pregunta creemos necesario remontarnos al contexto en el que surge el trabajo social. El mismo está condicionado por tres aspectos principales.

$1^{\circ}$. A lo largo de los siglos XIX y XX tiene lugar la expansión de las ciencias sociales. Como sabemos, a partir de la Revolución Francesa, las explicaciones dadas hasta ese momento sobre el funcionamiento de la sociedad fueron sustituidas por otras. Estas nuevas respuestas iniciaron el incipiente desarrollo de las ciencias sociales que hubieron de seguir el camino, el método y el modelo de las ciencias naturales para ser consideradas como ciencias. Creemos importante señalar que la noción de ciencia dominante (y única por aquellos momentos) gozaba de un lugar destacado en la sociedad como fuente de verdad. El conocimiento sólo se podía obtener a través de un procedimiento que pone 
el énfasis en lo objetivo, lo cuantitativo, lo experimental, la observación, la verificación y la generación de leyes de orden mecanicista (Naredo, 1987). La ciencia puesta al servicio del hombre y con la finalidad de dominar la realidad, social o natural. Si las ciencias sociales trataban de convertirse en ciencias y, para ello, se desarrollaron líneas de pensamiento por los precursores de las mismas, el trabajo social, por contra, nació como un saber empírico, como una actividad filantrópica (Zamanillo, 1991), centrado en la atención a los pobres en una mezcla de asistencia, control y represión. Sus preocupaciones «científicas» eran limitadas y se ceñían a cómo mejorar la organización de la caridad. Posteriormente, aparecería la necesidad del conocimiento de la realidad social, la profesionalización de la asistencia y su formación.

$2^{\circ}$. Las consecuencias sociales provocadas por la industrialización fueron muy importantes: no sólo porque motivaran la identificación del trabajador como pobre, sino porque fueron el motivo directo tanto del inicio de una tímida reformulación del liberalismo económico desde dentro como de propuestas más radicales desde el socialismo. Nos señala Rubio Lara (1991: 27-31) que en el siglo XIX se inició una revisión del orden socioeconómico implantado por el capitalismo liberal dada su patente injusticia. Revisiones que o bien se hacen desde el propio liberalismo de acuerdo a principios humanitarios y morales, o bien desde posturas más radicales que pretendían no reformar el sistema para asegurar su supervivencia, sino cambiarlo radicalmente (Marx). La sociedad no es vivida como un espacio de tranquilidad, seguridad y prosperidad, sino como un lugar de conflicto y tensión (Cotarelo, 1987). Desde las filas más conservadoras se desarrollaron acciones encaminadas a mejorar las condiciones de vida de los trabajadores y de los pobres (beneficencia, caridad). Con este deseo de mejorar las condiciones de vida y hacer aceptable el sistema (esto es, no cuestionarlo), se procederá a una revisión y expansión de las organizaciones de caridad, donde encontrará su origen el propio trabajo social. Las primeras visitadoras amigables, educadoras de la clase obrera, serán mujeres de la burguesía que pretenden mejorar su vida, guiada por el espíritu del utilitarismo (Moix: 1991, 57), que perseguía el mayor bien del mayor número. Quedaron en saco roto, de momento, las indicaciones emanadas de la Revolución Francesa que afirmará que la asistencia es una ciencia política que debe ser cuidadosamente estudiada (Álvarez Uría, 1986).

Si el impulso de las organizaciones de la caridad provino de las clases pudientes, no podemos obviar (por contraposición) que, en el siglo XIX, y gracias a la presión del movimiento obrero y de los partidos socialistas, comenzó también el desarrollado del Estado de Bie- 
nestar. Se podrá discutir si los logros alcanzados en este período, tanto en el orden político como social, son verdaderas conquistas de los trabajadores o, simplemente, cesiones del Estado y de la burguesía necesarias para el mantenimiento del sistema capitalista. En cualquier caso, lo que parece innegable es que la vida de los obreros empezó a mejorar, una redistribución incipiente de la riqueza se realizó y, sobre todo, se abrió definitivamente la puerta a la intervención del Estado en la regulación de la vida económica y en la protección y mejora de las condiciones de vida de los trabajadores. Esta intervención fue aceptada por los liberales como una manera de mantener el sistema y, por parte de los socialistas (al menos los lasellanos -Cotarelo, 1987-), fue el inicio de poner el Estado al servicio de la clase trabajadora. El incipiente Estado social nace a partir del reconocimiento de derechos a la clase obrera, el trabajo social nace con la finalidad de contribuir a mejorar las condiciones de vida de la misma, pero sin cuestionar la lógica que generaba las situaciones de enorme desigualdad: el objetivo era intervenir con medidas de previsión y protección social en el seno de la clase trabajadora, y ello no tanto en nombre del sacrosanto principio de la igualdad cuanto en nombre de la solidaridad, es decir, sin conceder a los asistidos derechos sobre el espacio político, espacio de soberanía (Álvarez Uría, 1995: 10-11). Si la clase obrera recibió bien el desarrollo de los primeros seguros sociales, recelaba frente a los primeros trabajadores sociales: unos eran vistos, aunque con discusiones, como conquistas de los trabajadores; otros eran percibidos como agentes de control.

$3^{\circ}$. El papel de la mujer en el siglo XIX también nos parece una referencia importante para conocer el contexto en el que nace el trabajo social. Su rol en la sociedad, la distribución de poderes, los espacios propios de los dos sexos, configurarán una presencia de la mujer en la sociedad subsidiaria del hombre, expulsada de la vida pública y cuya importancia, tanto en la producción como en la reproducción social, no es valorada suficientemente. Farge (1991: 90-96) nos señala que la mujer se caracteriza en este período, por estar sometida al hombre: $e l$ producto de su trabajo recae en su tutor legal y la procreación está sometida al control de la comunidad. Es considerada como un ser débil (y, por ello, quizás las primeras legislaciones de las condiciones de trabajo se dirigen a ellas y a los niños), lo que no impide que su trabajo siga siendo duro. Se les ve como irresponsables. Su ámbito de poder se limita a lo doméstico, donde se vive como una intrusión la presencia del hombre. En este espacio, tendrá lugar la socialización de hijos e hijas y, por tanto, la reproducción social. Lo único que cambia, según Farge, es que las mujeres (determinadas mujeres) comienzan a desarrollar una presencia pública que antes era de dominio exclusivo de los 
hombres. Veamos cómo lo dice la autora: las Iglesias, la República, exaltan "el poder social de las mujeres», considerado como fundamental en el desarrollo del Welfare Estate. En Alemania adopta la forma de una verdadera «maternidad social». En ese caso, las mujeres burguesas socorren, educan y controlan a las mujeres pobres y obreras. A impulso de asociaciones, pasan, de ser «visitantes de pobres» según la vieja expresión filantrópica, a inspectoras benévolas, de damas protectoras a asistentes sociales, precursoras de los trabajadores sociales... De la misma manera, los médicos hacen de las mujeres sus aliadas en la lucha por la higiene, que es también un modo de moralizar la miseria que se oculta tras la mugre. Muchas mujeres encontraban ahí un exutorio a sus energías y a la mala conciencia que los valores de utilidad y trabajo, crecientes en la sociedad, transmitían a su ociosidad (1991: 91).

Las mujeres se incorporan a la vida social progresivamente, siendo la asistencia social uno de los campos privilegiados para ello. Su presencia, su labor, es una prolongación de los roles expresivos que juegan en el ámbito doméstico y que reproducen en el ámbito público. Observamos también cómo el movimiento precursor del trabajo social nace en el seno de la clase alta y, a parte de servir para justificar a las mujeres frente a la sociedad, es empleado como un mecanismo de control respecto del cual la clase trabajadora recela, como había recelado con anterioridad de otros mecanismos de ayuda desarrollados por la sociedad (Fernando Díez, 1992: 116). La asistencia social, como precursora del trabajo social, nace pues de un deseo reformador desde las élites sociales, alejada del incipiente Estado social y sin participación en el debate sobre las ciencias sociales.

Entonces, ¿para qué una epistemología del trabajo social? ¿Puede mejorar la vida de la gente con las que intervienen los trabajadores sociales una construcción científica del trabajo social? Creemos que sí. Que el sujeto sea protagonista de su propio cambio, desarrolle una acción reflexiva, exige no sólo reconocerle un espacio en la relación, una influencia en el profesional, una capacidad en la toma de decisiones, requiere, por parte del profesional, de un conocimiento de la realidad y de su complejidad, que evite el interés técnico y se sitúe en las claves del interés emancipatorio.

\section{III.EN QUÉ CONSISTIRÍA ESTA EPISTEMOLOGÍA: CIENCIAS SOCIALES Y TRABAJO SOCIAL}

Las referencias interdisciplinares siempre han estado presentes en la profesión. Esta recurrencia ha sido conflictiva por dos razones prin- 
cipalmente: la obtención de los referentes teóricos en otras materias ha podido ser causa que explique el no desarrollo de la propia reflexión teórica y, en segundo lugar, por los criterios por los que dichos conocimientos se aplicaban al trabajo social: importaba más la orientación empírica, la resolución de un problema a partir del conocimiento tomado, que el ajuste del mismo al objeto, la naturaleza o los valores de la profesión. Esta adaptación de conocimientos supone una reducción del trabajo social a algunos de sus elementos fundamentales: su atención en lo psicológico, en lo individual, buscando la causa y la solución de los problemas en la propia persona. La atención principal en lo estructural, buscando, en este caso, la explicación y resolución de los conflictos únicamente en la sociedad, tensionada dialécticamente (Zamanillo, 1991: 37-43).

No obstante, recurrir a las ciencias sociales es una de las vías posibles para construir la disciplina: encontramos tanto elementos que nos ayudan a una justificación epistemológica del trabajo social como bases para el desarrollo de la disciplina. Quien piense que dicha relación es unidireccional, es decir, desde las ciencias sociales al trabajo social, se equivoca puesto que, tal y como demuestra Kam-Fong (1990), existen también aportaciones de nuestra profesión hacia ese ámbito más general que constituyen las ciencias sociales.

\section{1. ¿Qué podemos aprender de las ciencias sociales?}

$1^{\circ}$. Los modelos o paradigmas dominantes. Mardones y Ursúa (1982:15-35) nos plantean que, en el fondo del debate respecto a la consideración epistémica de las ciencias sociales, se encuentran dos tradiciones filosóficas sobre el método científico. La polémica entre ambas ha atravesado la historia de las ciencias sociales. La tradición aristotélica sostiene que en el principio de la investigación está la observación pero, que la explicación científica, no se logra hasta que no se dan razones de los hechos y fenómenos que son observados por el investigador. Por tanto, no es suficiente la observación. El investigador tiene que lograr una explicación de los hechos observados puesto que, sin tal explicación, no existiría conocimiento científico. El rasgo último y definitorio de esta corriente es la búsqueda de explicaciones teleológicas que aclaren las «causas finales»: se trata de buscar las razones que explican la existencia de los fenómenos. La tradición galileana defiende que las preocupaciones de la ciencia no son ya de orden metafísico sino funcional y mecanicista. Lo que interesa es buscar las leyes que explican el comportamiento de los fenómenos. El objetivo del conocimiento no se centra en saber el para qué o por qué de los hechos sino el cómo funcionan. Sólo así será posible establecer leyes científicas, expresadas matemáticamente, que expliquen los he- 
chos. Estas explicaciones funcionarán a modo de hipótesis causales que deberán experimentarse, ponerse a prueba.

$2^{\circ}$. Los condicionantes del conocimiento científico social. El objeto de conocimiento: lo social, el conjunto de relaciones interacciones, actuaciones e influencia de los hombres entre sí y con la sociedad institucional, la cultura y la naturaleza. Esta definición englobaría a un conjunto importante de campos de investigación: hechos externos al propio individuo que le influyen en su comportamiento, y hechos internos, motivados en el interior del individuo y que influyen también en su comportamiento (Sierra Bravo, 1984).

$3^{\circ}$. Las características peculiares de la realidad social. Vista bien desde el punto de vista de los sujetos que interaccionan o bien en su globalidad, se caracteriza por ser compleja, sutil y cambiante. Los fenómenos sociales se presentan a veces «tal como son» (realidad real) y, en otras ocasiones, su dimensión real está oculta, escondida (realidad aparente, que, a veces, puede ser real en la medida que existe como tal y, otras veces, esconde la realidad tal cual es) (Beltrán, 1982). Sin embargo, esta aproximación a la realidad no es compleja sólo por las características de la realidad social, sino también porque los objetos que en ella están no se presentan al investigador de manera inmediata, sino mediata, es decir, dicha aproximación está atravesada tanto por el todo social como por la experiencia previa del que se aproxima. Este conjunto de circunstancias determina un hecho fundamental: en la realidad social, objeto y sujeto forman parte de la misma al tratarse, como decía Ibáñez, de un sistema hiperreflexivo.

$4^{\circ}$. La relación objeto-sujeto y su incidencia en la objetividad. En el ámbito de las ciencias sociales ocurre que el sujeto que conoce ocupa, respecto al objeto conocido, una posición particular, puesto que el cognoscente forma parte de lo conocido. En cuanto que afecta a la objetividad, afecta a la validez del conocimiento científico social. Según Sierra Bravo (1984: 65), la objetividad la vamos a entender como la correspondencia del resultado del conocimiento con lo conocido. Como se trata de dos «productos» de naturaleza diferente -lo conocido es real mientras que el conocimiento es ideal-, la correspondencia no consiste en una reproducción de la realidad tal cual, sino que se concreta en términos y expresiones lingüísticas que designan los aspectos del fenómeno, sus propiedades y relaciones. Inciden en ella: el objeto sobre el que se conoce. En este caso, la realidad social, que es sensible, cambiante, múltiple, compleja. Este conjunto de rasgos determinan que los hechos sociales sean únicos. El sujeto, forma parte del objeto de conocimiento y, como ya hemos indiciado, puede ser causa o consecuencia de cambios en relación al objeto. Sobre la relación que el sujeto mantiene con el objeto se han desarrollado en las ciencias sociales 
diversas corrientes que enfatizan bien la objetividad, partiendo de parámetros positivistas y realistas, o bien la subjetividad, que se sostiene sobre el énfasis en el sujeto y en las representaciones mentales de éstos. Al respecto, Gualda Caballero (1996: 46-49) nos recuerda que tal polémica está superada por la reflexividad en la cibernética de segundo orden, que nos pone de manifiesto cómo el sujeto forma parte del objeto y está dotado de reflexividad: el sujeto observa al objeto, se observa a sí mismo y es observado por los objetos. El proceso de conocimiento que está influenciado por el sujeto y el objeto y la relación que mantienen entre sí.

$5^{\circ}$. La influencia de los valores en el conocimiento. Como parte del objeto, el sujeto que conoce puede poseer no sólo una experiencia previa o un conocimiento superficial, sino que puede valorar o apreciar los rasgos de los fenómenos sociales de acuerdo a su particular escala, puesto que toda persona posee valores que tienden a permanecer a lo largo del tiempo, lo que influye en su capacidad de conocer. Todos los fenómenos sociales pueden ser objeto de valoración por parte de las personas, aunque las jerarquías de valores varíen entre los grupos existentes en el seno de una sociedad (Duverger, 1981) y entre sociedades o culturas diferentes (Dumont, 1987).

$6^{\circ}$. El método en las ciencias sociales. ¿O los métodos? El seguir un método es condición imprescindible para que el conocimiento pueda ser científico. No obstante, como nos señala Bernal (1991), el método no ha sido algo fijo que no puede ser analizado sin tener en cuenta sus conexiones con el carácter social de la ciencia. Pensamos, con Feyerabend (1974), que los procedimientos utilizados por los científicos han sido diversos y que, por tanto, la práctica de los mismos no ha sido unánime en el uso del método científico. Al respecto, García Ferrando (1979: 50) nos pone de manifiesto que sí hay un acuerdo básico en cuanto a los principios de tal concepto: el pluralismo teórico, la dependencia contextual y teórica de la práctica científica, el recurso a la lógica y a la experiencia empírica, la metodología entendida como una actividad crítica y teóricamente dependiente... el acuerdo no va más allá de estos principios:... de aquí que... haya que escuchar con escepticismo las apelaciones, tan enfáticas como ruidosas, a un método científico riguroso, detallado, universal y «manualizable»: tal cosa, ciertamente, no existe. (Beltrán, 1991: 97-98).

En lo que afecta a las ciencias sociales, Beltrán (1991: 98) nos afirma que no deben mirarse en el espejo de las ciencias naturales por la peculiaridad de su objeto, completamente diferente puesto que es $s u b$ jetivo, en el sentido de que posee subjetividad y reflexividad propias, volición y libertad..., reactivo a la observación y al conocimiento... un objeto de una complejidad inimaginable. Este conjunto de caracterís- 
ticas determina necesariamente una pluralidad de metodologías para su conocimiento.

Los métodos que empleemos nos ponen de manifiesto una realidad, pero no la realidad tal y como es: nos permiten una aproximación a la misma pero mediatizada. Los métodos, como construcciones teóricas, están atravesados por concepciones previas que inciden en la realidad que ponen de manifiesto: seleccionan la realidad de alguna forma. Se impone un uso relativista de los mismos, siendo válidos en la medida que sean capaces de describir y explicar los hechos a los que se enfrentan. Más que una oposición de métodos, optamos por una complementariedad entre los mismos de acuerdo a la naturaleza de lo que se quiere estudiar.

$7^{\circ}$. Lo cuantitativo y lo cualitativo en las ciencias sociales. ¿Son incompatibles? Creemos que no. El debate entre ambos enfoques, siguiendo a Pérez Serrano (1994: 53), se produce en dos niveles diferentes: el primero de ellos, será el nivel epistemológico, donde se encuentran las mayores incompatibilidades entre ambos enfoques puesto que parten de concepciones diferentes acerca de la realidad social y de cómo abordarla. El encuadre en un paradigma u otro no supone necesariamente la elección en exclusiva de los métodos coherentes con el mismo. Es posible la combinación de métodos diferentes en función siempre de la naturaleza de lo que se quiere estudiar, de las exigencias planteadas en el estudio. El segundo nivel en el que se produce el debate es el nivel metodológico y técnico donde, a tenor de lo dicho hasta ahora, la incompatibilidad a priori es menor puesto que los problemas a resolver en este nivel aluden tanto a la rigurosidad del conocimiento obtenido como al empleo conjunto de ambos.

$8^{\circ}$. La concepción ampliada de las ciencias sociales y los intereses del conocimiento. Según Pourtois y Desmet (1992), la concepción ampliada podría ser el [camino] de la epistemología y de la metodología nuevas, a saber, aquél que favoreciera el análisis de las interacciones entre los individuos, el examen de la subjetividad del observado y del observador, la investigación de lo particular y del sentido, el tener en cuenta la dinámica de los acontecimientos, la historia de los individuos y la complejidad de los fenómenos

La concepción ampliada la contemplamos a partir de las orientaciones metodológicas resumidas en los siguientes puntos: subjetivi$d a d$, la importancia del material cualitativo que permite rescatar las actitudes, los valores, las opiniones, las percepciones, las creencias, las preferencias de los sujetos. La participación del observador: tener en cuenta la existencia del observador, sacar partido de la subjetividad inherente al acto de la observación y estudiar la reciprocidad de esta actividad. Significancia: la importancia de conocer los significados 
que los sujetos otorgan a los hechos que viven. Tiempo subjetivo: el tiempo de lo vivido. Los significados que los actores atribuyen a sus actos pueden variar, modificarse en función de la influencia de otros actores y/o acontecimientos.

Coherente con estas orientaciones metodológicas es la concepción del sujeto que se deriva de las siguientes características: Sujeto en interacción. La comprensión del sujeto no puede hacerse al margen del contexto en el que se ubica. Rasgos particulares: lo que pretende poner de manifiesto es la peculiaridad. Prevalece en esta concepción la singularidad. Diferencia: nos destaca la importancia de los contextos, de los entornos inmediatos a los sujetos a la hora de establecer los rasgos distintivos de los mismos. Complejidad. Los hechos sociales no tienen una única causa ni un solo determinante. Ambos son históricos y dinámicos. Se trata de comprender esta complejidad en la que cada elemento depende de los demás y sólo existe en ellos y por la relación de ellos. Comprender desde dentro, lo que nos remite de nuevo a tener en cuenta a los sujetos, a los significados que otorgan a los fenómenos sociales.

La construcción de las ciencias sociales no es ni objetiva ni neutra. Responde siempre a unos intereses que es necesario tener en cuenta para mantener una actitud crítica respecto al conocimiento. De los tipos de interés que identifica Habermas (1988) -interés técnico del conocimiento, interés práctico del conocimiento e interés de emancipación-, al trabajo social creemos que le corresponde este último, que está basado en la lógica de la comunicación, pero con una orientación «yo-yo», es decir, donde se potencia la capacidad de autorreflexión del sujeto. El investigador no domina, facilita que el sujeto sea capaz de autodeterminarse, de potenciar su autonomía propia. El objetivo, por tanto, se centra en la liberación del sujeto de las relaciones de dependencia que existen en su entorno. Es el interés propio de las ciencias sociales críticamente orientadas.

\subsection{El trabajo social como disciplina}

Para desarrollar este epígrafe consideramos importante hacernos tres preguntas: ¿cuál es la naturaleza del trabajo social (concepto). ¿De qué se ocupa? (objeto). ¿Cómo conoce dicho objeto (métodos)?

\subsubsection{Concepto de trabajo social}

Sobre la naturaleza de la disciplina se ha producido a lo largo de la historia un debate que todos conocemos. De las tres posturas mantenidas, arte, tecnología y ciencia, nos interesa destacar esta última. Klein 
y Bloom (1990) sostienen que la definición científica del trabajo social se apoya en cinco elementos: empirismo, que reúne tres tipos de datos: (1) la colección de datos cuantitativos primarios usando métodos empíricos estándares, como la investigación descriptiva y el análisis bivariante y multivariante; (2) la manipulación de los datos secundarios ya existentes, y (3) la investigación cualitativa, que incluiría los estudios de casos o la explicación de casos comunitarios, como un análisis de los datos secundarios o primarios, y los métodos etnográficos. Tecnología. Se refiere a la aplicación de los contactos empíricos a través de una amplia variedad de mecanismos. Conceptualización. Modo de pensar que se mueve de los acontecimientos específicos en el mundo cotidiano a un campo lógico y abstracto en donde las características seleccionadas se extraen y generalizan de manera que, esas entidades, se convierten en el objeto de nuestras consideraciones. Valoración. Toda actividad humana conlleva algún grado de elección preferencial (aunque sólo sea dedicarse a esa actividad y nada más). Con todo, como componente de una ciencia aplicada, la valoración tiene que ver con la presentación de una posición particular de estimación. Comentario. Se refiere a los estados que describen, critican o comentan las actividades empíricas, tecnológicas, conceptuales o de valoración.

Han sido necesarios muchos años y el avance epistemológico de las ciencias sociales para que las características del trabajo social que, vistas desde una perspectiva positivista no eran sino impedimentos en la construcción como ciencia de la disciplina, se revelaran como fortalezas (Méndez-Bonito, 1996), como amarras a las que asirnos en la construcción científica de la disciplina. Si estas características son la complejidad, unicidad, sensibilidad, el cambio..., la reflexión sobre las mismas en una perspectiva científica sólo era posible desde la concepción nueva o ampliada de las ciencias que, como ya vimos, nos proporcionan tanto orientaciones metodológicas que ponen el acento en la subjetividad, participación del observador, la significancia y el tiempo subjetivo, como una concepción del sujeto que es definido en interacción, en base a sus rasgos particulares, la diferencia y la complejidad. Se trata pues de construir conocimiento a partir de lo particular, de profundizar en algo concreto más que buscar la generalización.

\subsubsection{Objeto del trabajo social}

El objeto del trabajo social se localiza en la realidad social donde interaccionan los individuos entre sí y con los elementos presentes en la misma. Esta interacción determina la existencia de carencias, problemas, necesidades que requieren ser abordadas con un apoyo profesional, experto, para ser superadas. La cuestión es delimitar qué parte 
de esta realidad social y, más concretamente, qué espacio de la necesidad o problema corresponde al trabajo social. Al igual que ocurre con el todo social, creemos que el objeto de nuestra disciplina es complejo, dinámico y cambiante, que adquiere diversas manifestaciones y que, en consecuencia, debe ser abordado desde una pluralidad cognitiva y metodológica, siendo conscientes de que los diversos enfoques teóricos dirigen su conocimiento en una sentido poniendo de manifiesto un aspecto concreto de la realidad y no toda ella.

Creemos, con García Salord, que es necesario pensar y definir el objeto del trabajo social porque significaría deslindar analíticamente el objeto de intervención de los sujetos involucrados en la problemática, es decir, no nos corresponde como objeto toda la persona sino una de las dimensiones de la misma, lo que no significa que, para abordar la problemática concreta, no haya que tener en cuenta la multidimensionalidad del individuo. Ello supone identificar qué dimensión del problema social es la propia y específica del trabajo social para, posteriormente, distinguir la estructura del objeto a través de reconocer los elementos que lo componen y las interrelaciones establecidos entre ellos. Para ello, será fundamental considerar al trabajo social no desde una perspectiva práctica sino conceptual, aprehender nuestro objeto de trabajo y reflexionar sobre el mismo.

De la reflexión que hacemos sobre el objeto destacamos que la disciplina localiza su centro gravitatorio en la persona, ubicada siempre dentro de un contexto, interesándoles los aspectos subjetivos, desde el momento en el que se trabaja con individuos con autonomía para pensar y actuar, y los aspectos objetivos, ya que en la realidad social se dan situaciones sociales que tienen un carácter externo, «objetivo»y coercitivo con respecto al sujeto. Entre sujeto y contexto, entre las dimensiones objetivas y subjetivas, hay una continua influencia, pues si bien el contexto limita la posibilidad de acción de los sujetos, éstos modifican a aquél por medio de sus acciones (Hierro, 1996). Abordar estas dos dimensiones requiere de varias reflexiones: cómo es la realidad social y qué parte de la misma nos corresponde como disciplina, participamos de la que identifica el objeto como malestar psicosocial ordenado según su génesis estructural y su vivencia personal (Zamanillo,1992). Pensamos que se trata de una definición general, que se abstrae de lo inmediato, y que permite un abordaje conceptual del objeto. La definición del malestar viene establecida no sólo por la vivencia de una carencia, sino por la distancia que se establece entre la misma y la posibilidad de superarla obteniendo así una situación de bienestar. Al trabajo social le interesa conocer las situaciones de malestar en cuanto que los individuos pretenden mejorarla. La ayuda que se puede prestar en ese tránsito es lo específico de la profesión. Ahora 
bien, la definición del malestar necesita tener en cuenta el juego que se establece entre los individuos y entre éstos y la estructura social, es decir, necesita preguntarse cómo se genera y cómo se concibe este malestar. El malestar lo entendemos como una construcción históricosocial, fruto de la interrelación de los hombres, que genera situaciones de desigualdad, de desequilibrio interno en la propia interactuación y con la estructura social. Como producto social, la consideración de lo que se entiende por malestar está sujeta a cambios de acuerdo a coordenadas espacio temporales. No se entiende lo mismo por malestar psicosocial según el momento histórico y según la ubicación espacial. Igualmente, con la especialización del trabajo, con la movilidad estructural funcional (Zapatero, 1989) de bienes y personas que se produce en la ruptura del antiguo régimen y con la Revolución Industrial, la forma de hacer frente a ese malestar definido socialmente ha variado. Preguntarse por cómo se genera el malestar implica interrogarse cómo se le hace frente. Y es aquí donde aparece el trabajo social, como una forma especializada de ayuda (o control) a/de las personas que padecen dichas situaciones de malestar.

¿Cómo se construye el malestar? Nos dice Pelegrí (1995) que el objeto del trabajo social no es enteramente objetivo. Nosotros consideramos que es fundamentalmente ideológico en cuanto que subjetivo. La aproximación que hacemos al objeto está mediatizada por dos instancias:

- El todo social y los poderes públicos. Corresponde al conjunto de individuos en su interacción definir qué situaciones son conceptualizadas, son objetivadas, como malestar. Sin esta definición, no se legitima la intervención profesional que se realiza con ellas, es decir, no hay un espacio social para la misma. Desde esta consideración, la definición de una situación de malestar se convierte en un medio poderoso de control social puesto que determina quién puede recibir o no la ayuda, quién forma parte o no de la normalidad: seguimos posicionándonos ya haciendo juicios de valor ante eventos, circunstancias, hechos y situaciones, mediante pensamientos que no siempre hacemos explícitos pero que emergen... (Garcés Ferrer y Durá, 1998:52). Cuando existe el consenso en el seno de la sociedad, la intervención de los poderes públicos refrenda al mismo disponiendo y organizando una política de atención hacia el conjunto de personas que sufren la situación de malestar. En esta política encuentran los profesionales, entre ellos los trabajadores sociales, el paraguas que da sentido a su actuación. Ahora bien, como ya señalamos en la relación entre política social y trabajo social, las instituciones imponen a los profesionales objetivos, medios y fines pudiéndose originar conflictos entre estos y los de la 
profesión. Corresponde al trabajo social interrogarse cómo es esa definición social de malestar y las repercusiones que tiene para su ejercicio profesional.

- La segunda instancia es el propio trabajo social y los trabajadores sociales. Como miembros de la sociedad, los profesionales también interactúan con otros sujetos, con el todo social y con la estructura. Intercambian y comparten significados que contribuyen a crear los conceptos objetivados, entre ellos el de malestar social. Los trabajadores sociales se localizan en la parte normalizada, mayoritaria en la sociedad, en la que reside el poder de objetivación. Como consecuencia de ello, tiene una visión sobre los problemas y las manifestaciones materiales del objeto, del malestar. Esa visión, obtenida como miembro de la sociedad, es consustancial a su persona, a su ciudadanía, y de la que difícilmente se desprenden cuando intervienen como profesionales.

Si doble es el procedimiento de definición del objeto (en cuanto que construcción social y definición profesional), doble tiene que ser la devolución que se haga desde el propio trabajo social: hacia la sociedad a la que pertenece, influyendo en la configuración social del malestar, y hacia la propia profesión y disciplina, ayudando a establecer distancia sobre las opciones personales y reflexionando sobre la intervención en claves de subjetividad. Pensar sobre las mediaciones para ser conscientes de cómo construimos el malestar y cómo concebimos a quien lo padece. Pensar sobre las distancias para poder alejarnos de la racionalidad técnico instrumental y entrar en otra comunicativa y emancipadora.

\subsubsection{Los métodos en trabajo social}

Plantear la construcción de los métodos en trabajo social desde una perspectiva científica requiere de varias reflexiones, unas en el ámbito de las ciencias sociales y otras propias de nuestra disciplina. En primer lugar, ser conscientes del debate presente en el seno de las ciencias sociales sobre la existencia de un sólo método científico, que el mismo sea el de las ciencias naturales y que éste se pueda aplicar al conjunto de las ciencias sociales. Se trata de elegir un método, de acuerdo a la naturaleza propia de la parcela de la realidad que vayamos a estudiar, de los objetivos que persigamos con nuestra investigación y de las capacidades y medios del investigador. Sea cual sea el método elegido, no podemos renunciar a su carácter parcial, premeditado, en la medida que no nos pondrá de manifiesto toda la realidad social sino sólo una parte de la misma y desde unos planteamientos determinados. En segundo lugar, para la construcción científica del método en la discipli- 
na, es necesario tener en cuenta tanto las características peculiares de nuestro objeto como la relación objeto-sujeto que se da en trabajo social. En tercer lugar, el objeto de la disciplina participa de las características propias de la realidad social pero, al identificarse con el malestar psicosocial de los individuos, sobre su definición pesan mediaciones sobre las que es necesario establecer distancias para un abordaje conceptual de las manifestaciones materiales del mismo. En cuarto lugar, la construcción de los métodos en trabajo social requiere de un desde dónde se plantea tal construcción. Esta pregunta también ha quedado meridianamente respondida en nuestro trabajo. Las claves desde las que nos situamos nos remiten, por un lado, a la concepción ampliada de las ciencias sociales, desde la cual, las características del trabajo social se revelan como adecuadas y apropiadas para una construcción científica que respete la subjetividad, la participación del otro y la significancia como orientaciones metodológicas, y que concibe al sujeto en interacción, complejo, con rasgos particulares. El interés de emancipación de Habermas, con base en la lógica de la comunicación pero orientada hacia la autonomía y autorreflexión del sujeto.

Por estos condicionamientos epistemológicos, a la hora de abordar el método en trabajo social, tenemos que ser flexibles, respetando el carácter holístico de los fenómenos que giran en torno al malestar. Los métodos tendrán un carácter integral y globalizador, que contemple lo objetivo y subjetivo del objeto. Se trata de una metodología adaptada al objeto de estudio y de actuación, a los sujetos y a las circunstancias en que éstos se encuentran, que permita el conocimiento de los procesos de interacción social y de los comportamientos humanos. Entendemos pues que la pluralidad en los métodos debe ser la característica del trabajo social, dada la naturaleza de su objeto. La pregunta a plantear ahora es si el trabajo social tiene que crear métodos propios o puede realizar su construcción científica a partir de los métodos ya existentes. Ambas cuestiones creemos que no son incompatibles, pero, mientras llega la definición de los métodos propios, es decir, aquellos generados en el seno de la disciplina, el trabajo social debe trabajar con los métodos ya existentes, adaptándolos a su realidad y a sus peculiaridades.

La aproximación al conocimiento del objeto necesita de una doble estrategia que permita captar el contexto en el que se desarrolla y se manifiesta en una persona concreta. Las formas de acceder al conocimiento de ambas dimensiones apuntan a la necesidad de métodos y procedimientos cuantitativos y cualitativos. Los primeros, porque nos proporcionan un conocimiento del contexto, del entorno en el que se ubica la persona, aunque no son la única manera de acceder al conocimiento de lo contextual. En cuanto a los procedimientos cualitativos, 
su orientación más evidente se dirige hacia el conocimiento de la vivencia personal, a saber cómo la persona vive la situación concreta de malestar, qué significa y qué representa para ella. Los métodos cualitativos exigen una proximidad mayor entre trabajador social y cliente, entre profesional y lo que se ha definido como objeto de trabajo. No obstante, con estos procedimientos también podemos acceder al conocimiento de lo contextual en la medida que el discurso individual es también un producto social.

La conclusión general que podemos establecer es la necesidad de realizar investigaciones dentro del área de conocimiento que generen la teoría del trabajo social. Teoría que oriente tanto la práctica profesional propia de la disciplina como que constituya la aportación general al ámbito de las ciencias sociales. Investigaciones que mejoren la práctica profesional e investigaciones que versen sobre la propia disciplina. De acuerdo a las dos dimensiones del objeto, el sujeto y el contexto, los procesos de investigación a seguir se basarán tanto en la metodología cualitativa como la cuantitativa. Se trata de incorporar la investigación al campo de actuación del trabajo social. Campo de actuación que no se limita a la mera intervención profesional, sino que se extiende por la búsqueda de referentes teóricos que aludan a la propia disciplina y al contexto social donde ésta se desarrolla. No mejorar únicamente la práctica sino la presencia del trabajo social en el ámbito de las ciencias sociales. Esta práctica de la investigación requiere de la definición del objeto, de la selección de un o unos métodos y de su posterior desarrollo: un diseño, su implementación, una presentación de resultados y un sometimiento al juego de la contrastación, tanto de los resultados como de los procesos.

Pero la construcción del trabajo social no puede hacerse al margen de los nuevos vientos que corren en las ciencias sociales. Ya hemos dicho que nuestra profesión encuentra su lugar en la nueva concepción de las ciencias sociales y humanas. Una concepción dominada por una ruptura epistemológica con el paradigma positivista, lo que no supone un rechazo de los métodos cuantitativos. Creemos que esta construcción teórica pasa por la definición de una descripción densa (como la entiende Geertz -1992-) para el trabajo social. Un esfuerzo intelectual que nos permita ver, en las interactuaciones cara a cara con los individuos, distintos niveles de significación: desde aquél que se sitúa en lo más evidente, superficial, hasta el que se localiza en la estructura profunda y que dota de significado a los comportamientos individuales. En una intervención directa, los trabajadores sociales se enfrentan a gran parte de los problemas que abordan las ciencias sociales. Basta con que tengamos la posibilidad de traspasar lo evidente para descubrir todo un mundo de significados complejos que necesitan de un en- 
tendimiento y de una confrontación para poder ser comprendidos. Interrogarnos sobre por qué el otro se comporta de una determinada manera, qué significa para él dicha actuación, qué significa para nosotros..., nos coloca en la tesitura de estructurar un conocimiento autónomo.

\section{BIBLIOGRAFIA}

ÁLVAREZ URÍA, F.: «Los visitadores del pobre. Caridad, economía social y asistencia en la España del siglo XIX», en VARIOS AUTORES: De la beneficencia al bienestar social. Cuatro siglos de historia de la acción social. Siglo XXI. Madrid, 1986.

ÁVAREZ URÍA, F.: «En torno a la crisis de los modelos de intervención social», en VARIOS AUTORES: Desigualdad y pobreza hoy. Talasa. Madrid, 1995.

BELTRÁN, M .: «La realidad social como realidad y apariencia». En REIS, $\mathrm{n}^{\circ}$ 37, 1982, pp. 25-53.

BELTRÁN, M.: La realidad social. Tecnos. Madrid, 1991.

BERNAL, J.D.: Historia social de la ciencia. Península. Barcelona, 1991.

FARGE, A.: «La historia de las mujeres. Cultura y poder de las mujeres: ensayo de historiografía». En Historia Social $n^{\circ}$ 9: historia de las mujeres, historia del género, 1991, pp. 79-101.

FERNANDO DÍEZ, R.: «Estructura social y sistema benéfico-asistencial en la ciudad preindustrial». En Historia Social $n^{\circ}$ 13: Pobreza y asistencia social, 1992, pp. 101-121.

FEYERABEND, P.: Contra el método. Ariel. Barcelona, 1974.

GARCÍA COTARELO, R.: «Origen y desarrollo del Estado de Bienestar». En Sistemas n ${ }^{\circ}$ 80/81. 1987, pp. 5-22.

GARCÉS FERRER, J. y DURÁ, E.: «Ideología y política social». En GARCÉS FERRER, J. y ALEMÁN BRACHO, C. (Coord.):Política social. Mc Graw Hill. Madrid, 1998, pp. 51-77.

GARCÍA FERRANDO, M.: «La Sociología, ¿una ciencia multiparadigmática?, en JIMÉNEZ BLANCO, J. y otros (comps.): Teoría sociológica contemporánea. Tecnos. Madrid, 1978.

GARCÍA SALORD, S.: Especifidad y rol en trabajo social: curriculum, saber, formación. Humanitas. Buenos Aires, 1991.

GEERTZ, C.: La interpretación de las culturas. Gedisa. Barcelona, 1992.

GUALDA, E.: «Pluralismo metodológico y cognitivo y articulación de técnicas». En CRUZ, F. y GUALDA, E. (Coord.): Métodos y técnicas de investigación social. Asociación Miguel Ángel Caro. Huelva, 1996. 
HABERMAS, J.: Conocimiento e interés. Taurus. Madrid, 1988.

HIERRO, M.: Proyecto Docente para concursar a una plaza de profesora titular de escuela universitaria. Área de trabajo social y servicios sociales. Material inédito, 1996.

KAN-FONG MOINT CHEUNG: «Interdisciplinary relationships between social work and other disciplines: a citation study». En Social Work Research and Abstracts, volumen 26, $\mathrm{n}^{\circ} 3,1990$, pp. 23-29.

KLEIN, W \& BLOOM, M. (1990): «Social work as applied social science: A historical analysis. En Social Work, volumen 39, n 4, 1990, pp. 421-431.

MARDONES, J.M. y URSÚA, N.: Filosofía de las ciencias humanas y sociales. Fontamara. Colección Logos. Barcelona, 1982.

MÉNDEZ-BONITO ZORITA, P.: «El trabajo social en sociedades complejas en el contexto de una modernidad en crisis». En Ponencias y comunicaciones oficiales al VIII Congreso Estatal de diplomados en Trabajo Social y asistentes sociales. Consejo General de Diplomados en Trabajo Social y asistentes sociales. Sevilla, 1996, pp. 139-148.

MOIX MARTÍNEZ, M.: Manual de Trabajo Social. Trivium. Madrid,1991.

NAREDO, J.M.: La economía en evolución. Historia y perspectivas de las categorías básicas del pensamiento económico. Siglo XXI. Madrid, 1987.

PELEGRÍ, X.: «La relación de los profesionales del trabajo social con su objeto de trabajo». En RTS n 137, 1995, pp. 87-100.

PÉREZ SERRANO, G.: Investigación cualitativa. Retos e interrogantes. I. Métodos. La Muralla. Madrid, 1994.

POURTOIS, J.P. y DESMET, H.: Epistemología e instrumentación en ciencias humanas. Herder. Barcelona, 1992.

RUBIO LARA, M.J.: La formación del Estado Social. Ministerio de Trabajo y Seguridad Social. Madrid, 1991.

SIERRA BRAVO, R.: Ciencias sociales. Epistemología, lógica y metodología. Teoría y ejercicios. Paraninfo. Madrid, 1984.

ULISES MOULINES, C.: «Epistemología». En REYES, R (ed.):Terminología Cientifico social. Anthropos, pp. 347-350. Barcelona, 1988.

ZAMANILLO PERAL, T.: «La evidencia empírica». En ZAMANILLO, T. y GAITÁN, L.: Para comprender el trabajo social. Verbo Divino. Navarra, 1991.

ZAMANILLO PERAL, T.: «La intervención profesional». En VII Congreso Estatal de diplomados en Trabajo Social y asistentes sociales. Barcelona, 3, 4 y 5 de diciembre de 1992. 